\title{
Study of dielectric properties of nanophase silver iodide
}

\author{
M ABDULKHADAR* and BINNY THOMAS \\ School of Pure and Applied Physics, Mahatma Gandhi University, Priyadarshini Hills P.O., \\ Kerala 686560 , India
}

MS received 5 September 1995; revised 11 March 1996

\begin{abstract}
Dielectric properties of nanophase AgI are studied over the frequency range from $100 \mathrm{kHz}$ to $3 \mathrm{MHz}$ at different temperatures. The values of $\varepsilon$, $\tan \delta$ and $\sigma_{\mathrm{ac}}$ are considerably larger than those reported for crystalline pellets of AgI, but they show a similar trend in variation with frequency and temperature. The increase in these values are attributed to the defect structure of the nano-particles.
\end{abstract}

Keywords. Nanoparticles; dielectric properties.

\section{Introduction}

In the past few years, atom clusters with average diameters in the range of 5-50 $\mathrm{nm}$ of a variety of materials have been synthesized and consolidated to create nanophase materials (Andres et al 1989). These materials have properties that are often significantly different and considerably better relative to those of their bulk counterparts. Since their properties can be engineered during the synthesis and processing steps, cluster-assembled nanophase materials appear to have great technological potential (Siegel 1994).

It would be of particular interest to investigate how the conductivity of the ionic conductor gets affected with reduction in the size of the crystal to nano-size range. More important would be to make such a study in super-ionic conductors. Superionic conductors or solid electrolytes characterized by exceptionally high ionic conductivity and relatively small electronic conductivity are materials of great technological interest due to their potential application in solid state batteries and other devices (Chandra 1981). The dc conductivity of nano-phase AgI over a temperature range covering the stability range of $\beta$-phase of $\mathrm{AgI}$ and well beyond the $\beta$ to $\alpha$ transition temperature has been reported recently (Abdulkhadar and Binny Thomas 1994). The present paper reports the study of dielectric properties of nanophase AgI, compacted into pellets, over a wide frequency and temperature range.

\section{Experimental}

The nano-particles of AgI in the present study were prepared by chemical precipitation from aqueous solution of $\mathrm{AgNO}_{3}$ and $\mathrm{KI}$ as reported earlier (Abdulkhadar and Binny Thomas 1994). The crystal structure of the particles was determined by X-ray powder diffraction using a SHIMADZU XD610 powder diffractometer and was found to be $\beta$-phase (wurtzite). The average size of the particles determined by the diffraction line-broadening making use of Scherrer's (Taylor 1961) equation was found to be $18 \mathrm{~nm}$. The particles were pressed into pellets of diameter $13 \mathrm{~mm}$ and

*For correspondence 

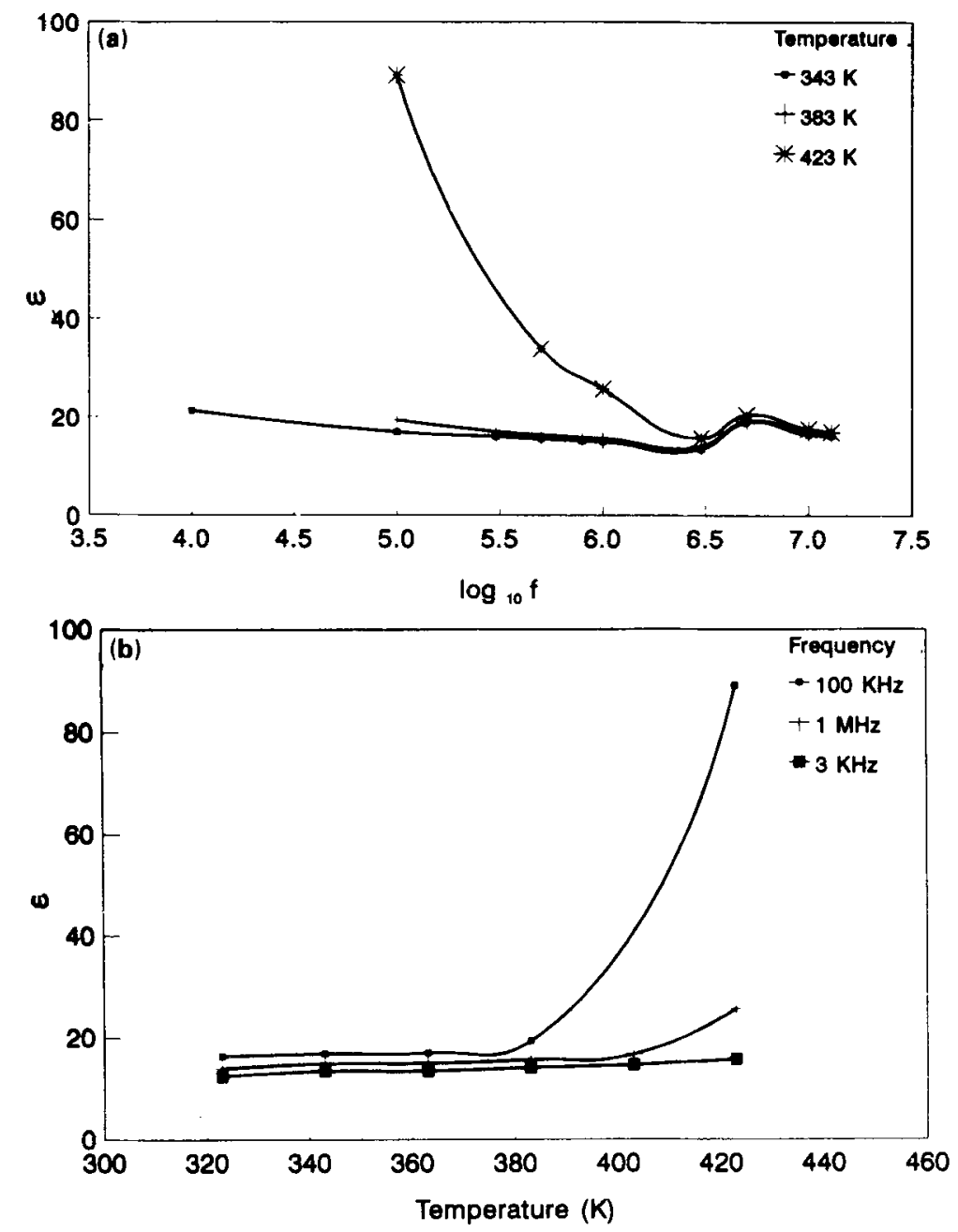

Figure 1. a. Variation of dielectric constant with frequency of nano-particles of AgI and b. variation of dielectric constant with temperature of nano-particles of Agl.

thickness $2 \mathrm{~mm}$ at a pressure of 4 tonnes $/ \mathrm{cm}^{2}$ for the dielectric measurements. Dielectric constant $(\varepsilon)$, dielectric loss $(\tan \delta)$, and ac conductivity $\left(\sigma_{\mathrm{ac}}\right)$, of the samples were determined using a HEWLETT PACKARD Model 4192A LF Impedance Analyzer over a frequency range from $100 \mathrm{kHz}$ to $3 \mathrm{MHz}$ and at different temperatures from 300 to $500 \mathrm{~K}$.

\section{Results}

The variation of $\varepsilon$ as a function of frequency and temperature are shown in figures 1a and $b$ respectively. It was found that at a temperature $343 \mathrm{~K}, \varepsilon$ had a value of about $21 \cdot 3$ at $10 \mathrm{kHz}$ and it decreased with frequency at a low rate, reached a minimum value of 13.4 at about $3 \mathrm{MHz}$, then there was a slight up and down variation with frequency. The variation of $\varepsilon$ with frequency showed the same trend at different temperatures, $\varepsilon$ at 

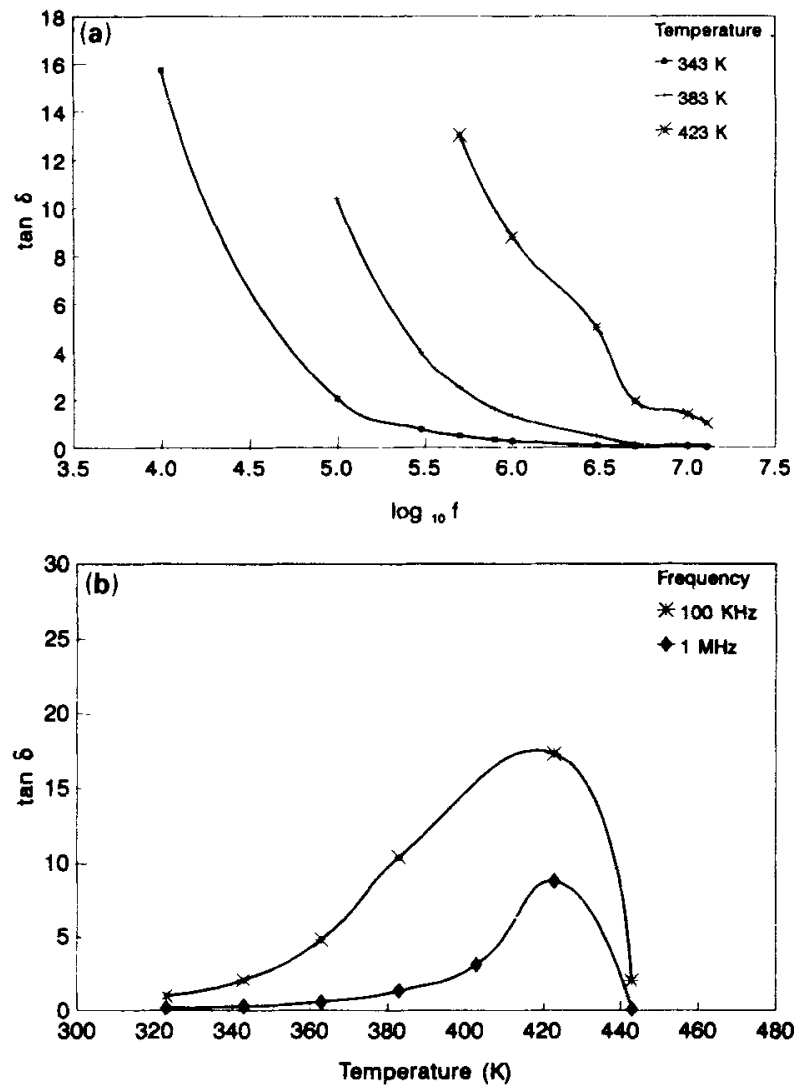

Figure 2. a. Variation of dielectric loss with frequency of nano-particles of AgI and b. variation of dielectric loss with temperature of nano-particles of AgI.

$423 \mathrm{~K}$ decreasing rapidly with frequency from a large value of about 89 at $100 \mathrm{kHz}$ to 15 at $3 \mathrm{MHz}$. At a constant frequency, $\varepsilon$ increased slowly with temperature initially and then increased rapidly, reaching a value of about 89 and 25 at $423 \mathrm{~K}$ and respectively at $100 \mathrm{kHz}$ and $1 \mathrm{MHz}$.

Figure 2a shows that $\tan \delta$ decreased rapidly with frequency tending to attain a low value at high frequencies above $1 \mathrm{MHz}$. From figure $2 \mathrm{~b}$ can be seen the variation of $\tan \delta$ with temperature: $\tan \delta$ increased with temperature at a slow rate initially, then reached a large maximum value at a rapid rate and then decreased.

$\sigma_{\mathrm{ac}}$ at different temperatures was almost independent of frequency, while it increased with temperature. The $\sigma_{\mathrm{ac}}$ vs $1000 / T$ graphs corresponding to different frequencies merge together (figure $3 b$ ). The curve corresponding to higher frequencies showed that $\sigma_{\mathrm{ac}}$ increases with temperature at a fairly constant rate with an activation energy of $0.55 \mathrm{eV}$ up to about $403 \mathrm{~K}$, then underwent an enhancement by about one order of magnitude over a temperature range of $\sim 10 \mathrm{~K}$. The numerical value of $\sigma_{\mathrm{ac}}$ at $1 \mathrm{MHz}$ increased by about 2 orders of magnitude from a value of $1.35 \times 10^{-4}{\mathrm{~S} . \mathrm{cm}^{-1}}^{-1}$ at $323 \mathrm{~K}$ to a value of $1.25 \times 10^{-2} \mathrm{~S} . \mathrm{cm}^{-1}$ at $423 \mathrm{~K}$ compared to a value of $\sim 10^{-6} \mathrm{~S}^{-\mathrm{cm}^{-1}}$ at about $294 \mathrm{~K}$ and to a value of $\sim 10^{-4} \mathrm{~S} . \mathrm{cm}^{-1}$ at about $453 \mathrm{~K}$ reported for crystalline pellets of AgI (Gon and Rao 1980). 

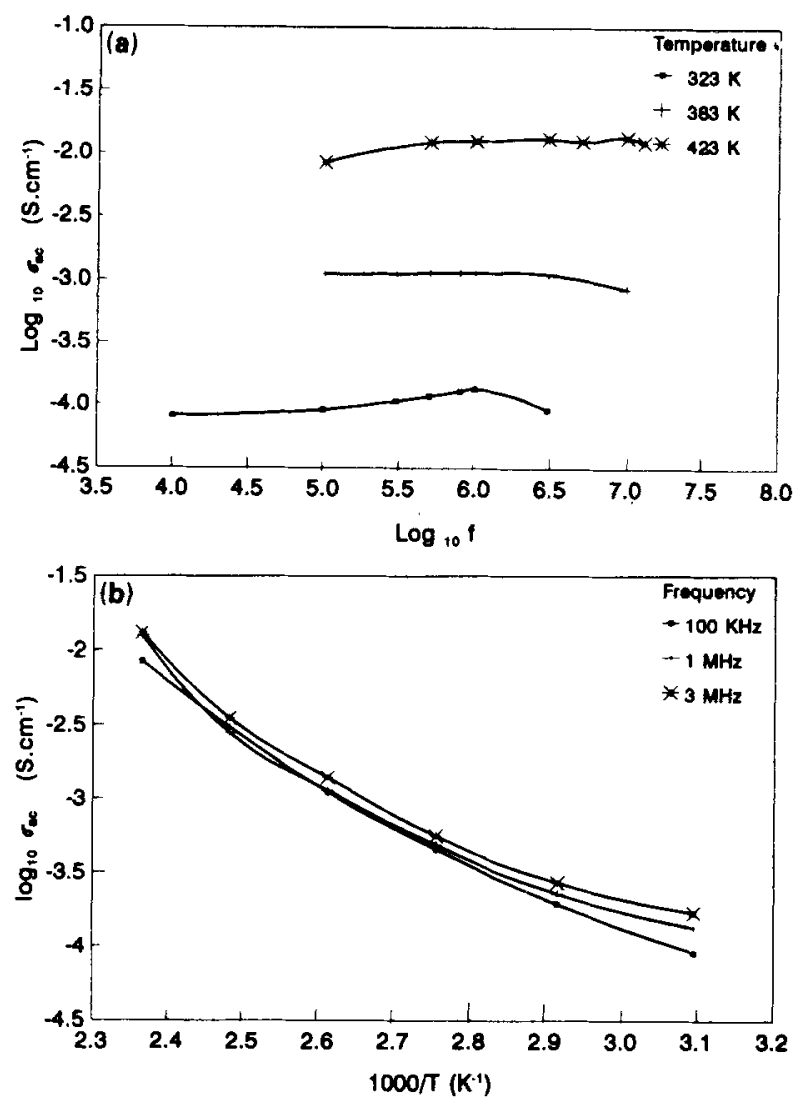

Figure 3. a. Variation of $\rho_{\mathrm{ac}}$ with frequency of nano-particles of $\mathrm{AgI}$ and $\mathbf{b}$. variation of $\sigma_{\mathrm{ac}}$ with temperature of nano-particles of $\mathrm{AgI}$.

\section{Discussion}

In the case of crystalline pellets of AgI, the value of dielectric constant $(\varepsilon)$ at temperatures close to room temperature $(303 \mathrm{~K})$ and at low frequencies has been reported (Cochrane and Fletcher 1971; Gon and Rao 1980) to be less than 20 which decreased with frequency and attained a frequency independent value of 7 at $1 \mathrm{MHz}$. It is also reported that $\varepsilon$ increased with temperature at a slow rate up to about $373 \mathrm{~K}$ and rapidly above this temperature, the rate of increase at higher temperatures being highly frequency dependent, and attained a value of about 35 and 15 at $423 \mathrm{~K}$ and respectively at frequencies of $100 \mathrm{kHz}$ and $1 \mathrm{MHz}$. These studies showed that the dielectric loss $(\tan \delta)$ had a value less than 10 at low temperatures and at low frequencies, which decreased with increase in frequency to a low value of about 0.07 at $10 \mathrm{MHz}$. Tan $\delta$ at low frequencies and at low temperatures in the range of 193 to $340 \mathrm{~K}$ increased with increase in temperature, reaching a maximum value of $\approx 10$ and thereafter decreased (Cochrane and Fletcher 1971).

In the present study $\varepsilon$ is found to have a value of 21.3 at $343 \mathrm{~K}$ and at $10 \mathrm{kHz}$ which decreases to a value of 16.2 at $13 \mathrm{MHz}$ (figure 1a). The variation of $\varepsilon$ with temperature is found to exhibit the same trend as reported (Gon and Rao 1980; Cochrane and Fletcher 
1971) (figure 1b) viz. $\varepsilon$ increases with temperature. the rate of increase being frequency dependent. $\varepsilon$ is found to attain values of about 89 and 25 at $423 \mathrm{~K}$, and respectively at frequencies of $100 \mathrm{kHz}$ and $1 \mathrm{MHz}$. Thus the high frequency dielectric constant $(1 \mathrm{MHz})$ at low as well as high temperatures is found to have a larger value than the values reported for crystalline pellets (Gon and Rao 1980). The dielectric constant of pellets of crystalline AgI at low frequencies has been ascribed to space charge polarization due to charge defects (Gon and Rao 1980). It is well known that the crystal structure of the surface layers of nanometer-sized particles is different from that of the core and the surface layers are often amorphous-like (Hayashi et al 1982; Hayashi and Abe 1984). Since in the case of very small particles, the surface layers form a larger part of the total volume of the particles, the structural anomaly of the surface layer is bound to play a prominent role in the physical properties exhibited by individual as well as collection of these particles. In the present study, the dielectric constant of the nano-particles which decreases only slightly with frequency may be attributed to ion jump polarization (orientation polarization) in addition to space charge effects. Due to the defect structure of the individual particles there will be a number of interstitial positions for the ions to jump to. Ion jump polarization effects occur in solids when two or more equivalent positions for an ion are present. At high temperatures, there is an increasing contribution to the polarization resulting from ion mobility and crystal imperfection mobility (Kingery 1976).

It has been reported that at high temperatures dc conductivity of AgI nano-particles increased by about 2 orders of magnitude and the activation energy of the mechanism of conduction is a function of temperature (Abdulkhadar and Binny Thomas 1994). This enhancement has been attributed to factors which depend on the small size of the AgI particles which influence the number of interstitial ions and their mobility. The nano-particles may be considered to be in a defect structure (Solliard 1985) which may cause an increase in the space charge as well as ion jump polarizations. Moreover at high temperatures dc conductivity effects, which increase exponentially with temperature becomes important. The combined effect is to produce a sharp rise in the dielectric constant with increasing temperature at low frequencies, corresponding to both ion jump orientation effects and space charge effects.

It is known that electronic and ionic polarizations are not affected significantly by temperature in the temperature range of the present study. In addition, even if we assume that a small number of dipoles are present and they contribute to enhance the dielectric constant $\varepsilon, \varepsilon$ should decrease with temperature since according to Debye's theory $\varepsilon$ is inversely proportional to temperature (Suryanarayana et al 1984). As such $\varepsilon$ of $\mathrm{AgI}$ should not change considerably with increase in temperature. However, it has been reported (Gon and Rao 1980) in the case of bulk AgI that $\varepsilon$ increases rapidly with temperature ( $\varepsilon$ being about 5 times at $423 \mathrm{~K}$ compared to the value at $323 \mathrm{~K}$ ), the increase being considerably frequency dependent. This behaviour has been attributed to space charge polarization due to defects and in part due to ionic polarization. In the present study, $\varepsilon$ is found to increase only by about $25 \%$ at $100 \mathrm{kHz}$ and by about $100 \%$ at $1 \mathrm{MHz}$ over the temperature range from 327 to $427 \mathrm{~K}$. This shows that even though the increase in $\varepsilon$ may be attributed to space charge and ionic polarizations, the small size of the particles has prevented the increase in polarization so that the rate of increase in $\varepsilon$ value is much less than that reported for crystalline pellets of Agl. The larger value of $\varepsilon$ at high frequencies and at low as well as high temperatures compared to the corresponding values reported in the literature (Cochrane and Fletcher 1971; Gon and 
Rao 1980) may be ascribed to peculiar properties of the nanophase, which are yet to be fully understood.

Tan $\delta$ in the present study is found to have a larger value than that of the pellets of crystalline AgI (Gon and Rao 1980) and it decreases more rapidly tending to attain a constant value indicating that $\tan \delta$ is inversely proportional not to the frequency $f$, but to some power of $f$ (figure $2 \mathrm{a}$ ). The variation of $\tan \delta$ with temperature exhibits a behaviour very close to that reported for pellets of crystalline AgI samples, but the peak value of $\tan \delta$ is attained at a higher temperature than that reported (Cochrane and Fletcher 1971) (figure 2b). The rapid decrease of $\tan \delta$ with frequency is again attributable to the change in the processes responsible for energy absorption in nano-particles compared to those in bulk crystals. The presence of a peak in the $\tan \delta \mathrm{vs}$ temperature graph must be connected to the crystal structure transition from $\beta$ phase to $\alpha$ phase which is expected around $420 \mathrm{~K}$. The rapid decrease in $\tan \delta$ with frequency (figure 2a) tending to a frequency independent value indicates that ion jump and dipolar relaxation processes are responsible for the dielectric loss and that the small size of the particles influences these processes reducing $\tan \delta$ rapidly.

The ac conductivity measurements of bulk samples of AgI (crystals as well as pellets of polycrystalline materials) have been reported to exhibit two regions (Biermann and Jost 1960; Funke 1976): (i) the impurity region at low temperature and (ii) intrinsic conduction region beyond about $373 \mathrm{~K}$. The moderately large value of activation energy $(0.55 \mathrm{eV})$ in the intrinsic region suggests that the conductivity in the region $373-473 \mathrm{~K}$ is due to $\mathrm{Ag}^{+}$ ion vacancy movement (Gon and Rao 1980). In the present study $\sigma_{\mathrm{ac}}$ is found to be only weakly dependent on frequency (figure $3 a$ ) but it shows an increase with temperature (figure $3 \mathrm{~b}$ ). Also the value of conductivity is 2 to 3 orders of magnitude larger than that reported for crystalline pellets of AgI. The absence of a sharp temperature for the transition from the $\beta$ phase to $\alpha$ phase (figure $3 \mathrm{~b}$ ) and the lack of a sharp increase in conductivity as a result of this transition indicates that in nano-particles the processes of conduction as well as the phase transition are effectively modified compared to those in bulk crystals. The observation that even at low temperatures the conductivity is about two orders of magnitude larger than that of pellets of crystalline AgI (Gon and Rao 1980) supports the reasoning that the nano-particles are in a defect state (Solliard and Flueli 1985 ) enhancing the conductivity and altering the polarization processes which account for the observed changes in $\varepsilon$ and $\tan \delta$.

\section{Conclusion}

The dielectric properties of consolidated nano-particles of $\mathrm{AgI}$ at different frequencies have been studied over a large temperature range. $\varepsilon, \tan \delta$ and $\sigma_{\mathrm{ac}}$ are considerably larger than the reported values for crystalline pellets of AgI, even though they show a similar trend in variation with frequency and temperature. The observed changes in the dielectric properties are attributed to the defect structure of the nanophase AgI.

\section{References}

Abdulkhadar M and Binny Thomas 1994 Solid state ionic materials (eds) B V R Chowdari et al (Singapore: World Scientific Publishing Co) p. 247

Andres R P et al 1989 J. Mater. Res. 4704

Biermann W and Jost W 1960 Z. Phys. Chem. (Fankf a.M) 17139 
Chandra S 1981 Superionic solids (New York: North Holland Publishing Co)

Cochrane G and Fletcher H N 1971 J. Phys. Chem. Solids 322557

Funke K 1976 Prog. Solid State Chem. 11345

Gon H B and Rao K V 1980 Indian J. Phys. 54A 50

Hayashi S, Ito M and Kanamori H 1982 Solid State Commun. 4475

Hayashi S and Abe H 1984 Jpn J. Appl. Phys. 23 L824

Kingery W D 1976 Introduction to ceramics (New York: John Wiley) 2 ed. p. 938

Siegel R W 1994 Physics of new materials (ed.) F E Fujita (New York: Springer Verlag) p. 65

Solliard C and Flueli M 1985 Surf. Sci. 56487

Suryanarayana P, Acharya H N and Rao K V 1984 J. Mater. Sci. 321

Taylor A $1961 X$-ray metallography (New York: Wiley) p. 674 